and condition-specific GItools could make efficient use of resources.

\section{PUBMED VS. GOOGLE SCHOLAR: A DATABASE ARMS RACE?}

M Thiese, A Effiong, D Passey, U Ott, K Hegmann. University of Utah Rocky Mountain Center for Occupational and Environmental Heal, Salt Lake City, USA

\section{0:1136/bmjgs-2013-002293.96}

Background Currently there are two widely used databases, PubMed and Google Scholar, are used for guidelines development. Research suggests PubMed is superior, however recent evidence suggests Google Scholar may have closed that gap. One family of journals reports $60 \%$ of their traffic is coming from Google Scholar.

Objectives Assess efficiency and completeness of searching for known moderate and high quality RCTs in PubMed and Google Scholar.

Methods Searches were performed by two experienced researchers using the same search terms to identify RCTs for a specific treatment. In a crossover design, one researcher performed the search in PubMed (PM1), the other in Google Scholar (GS1). Subsequently each performed the same searches in the other database (PM2 and GS2). Total numbers of articles identified, relevant articles found, and the time to complete were collected. Articles were compared to a known comprehensive list of 5 RCTs used for guideline preparation that was drawn from 6 exhaustive database searches.

Results GS1 identified 2 and GS2 identified 3 of the RCTs. PM1 identified 2 and PM2 identified 2 RCTs. PubMed and Google Scholar searches averaged 63 and 194 minutes to complete respectively.

Discussion Each database consistently identified one of the two highest quality studies, but neither database identified both. Difference search time is nearly 3 -fold. No single search identified all quality studies. Additional trials are planned.

Implications for Guideline Developers/Users For comprehensive literature searches both databases should be searched.

\section{HOW ARE WE FEELING TODAY? THE SENSITIVITY OF A LITERATURE SEARCH FILTER FOR PATIENTS' VALUES AND PREFERENCES}

${ }^{1} \mathrm{M}$ Wessels, ${ }^{2} \mathrm{~L}$ Hielkema. ${ }^{1}$ Knowledge institute of Medical Specialists (KiMS), Utrecht, The Netherlands; ${ }^{2}$ Dutch College of General Practitioners (NHG), Utrecht, The Netherlands

\section{0:1136/bmjqs-2013-002293.97}

Background The patient perspective in guideline development is of vital importance. To find out what this perspective entails, different methods may be considered, such as participation of patients or their representatives in guideline development groups or in focus group discussions, or by conducting patient surveys addressing specific problems and needs. In addition, a review of the literature in the early stages of guideline development can provide relevant information. Literature search filtres for patients' perspectives and preferences applicable for Medline (OVID), PubMed, and Embase were developed and validated in 2012. The specificity was $98 \%$ but the sensitivity was only $90 \%$. Objectives To verify the sensitivity of the filtres by means of a newly available 'gold standard'.
Methods We re-estimated the sensitivity of the search filtres by using the references of a recent Cochrane Review, Interventions for providers to promote a patient-centred approach in clinical consultations 2012;(12):CD003267, as a gold standard.

Results The search filtres for patients' values and preferences retrieved 72 (Medline (OVID/Pubmed) and 67 (Embase) titles, respectively, out of 73 references included in the Cochrane Review (mean sensitivity 96\%).

Discussion Applying filtres for patients' perspectives and preferences retrieved almost all references. Minor adaptations to the Embase filtre were needed to enhance the sensitivity without compromising the specificity. Validation of filtres is an iterative process, illustrating that filtres are dynamic tools.

Implications for Guideline Developers/Users Availability of a validated tool for retrieving literature on patients' values and preferences can support integration of the patient perspective in guideline development.

\section{CHALLENGES OF DEVELOPING RAPID GUIDANCE FOR COMPLEX INTERVENTIONS}

J Franek. Southern California Permanente Medical Group, Los Angeles, United States

\section{0:1136/bmjqs-2013-002293.98}

Background Rigorous guideline development requires extensive time and resources. Rapid review-a streamlined approach to synthesising evidence-offers an attractive alternative to systematic review for informing decision-making on complex interventions in a timely manner. Complex interventions are those that contain extensive number of interacting components.

Context A rapid evidence assessment service of a large US-based health care organisation developed guidance through rapid review on transitional residential recovery services (TRSS) for substance abusers.

Description of Best Practice Complex interventions present unique challenges for evaluation by rapid review. Significant scoping and upfront communication with end users was undertaken to understand the target populations, intervention-related components, outcomes, timing and settings associated with TRSS. Thorough refinement of Ovid search algorithms using date-based limits was needed to generate a feasible and appropriate literature database. Issues relating to complex interventionssuch as limited generalisability, lack of effect may be driven by poor implementation rather than ineffectiveness of intervention, variability in outcomes, etc.-were communicated to end users in conjunction with findings. Changes to existing programmes were enacted based on findings and will be discussed.

Lessons for Guideline Developers, Adaptors, Implementers, and/or Users Studies of complex interventions are notoriously difficult to evaluate and summarise through traditional evidence assessment methods. Rapid review offers an attractive option for providing evidence for timely decision-making; however, its application to complex interventions requires careful planning, execution and understanding.

\section{INTEGRATING GUIDELINES INTO LOCAL CLINICAL PRACTICE AND POLICY USING HOSPITAL-BASED HEALTH TECHNOLOGY ASSESSMENT}

${ }^{1,2} \mathrm{M}$ Mitchell, ${ }^{1,2} \mathrm{~B}$ Leas, ${ }^{1,2} \mathrm{~J}$ Lavenberg, ${ }^{1,2,3} \mathrm{~K}$ Williams, ${ }^{1,2,4,5,6,7} \mathrm{C}$ Umscheid. ${ }^{1}$ Center for Evidence-based Practice, University of Pennsylvania Health System, Philadelphia, USA; ${ }^{2}$ ECRI Institute-Penn Medicine Evidence-based Practice Center, Philadelphia, USA; 